\title{
Nonreflecting Boundary Conditions For Electromagnetic Scattering
}

\section{Report}

Author(s):

Grote, Marcus J.

Publication date:

1998-09

Permanent link:

https://doi.org/10.3929/ethz-a-004284976

Rights / license:

In Copyright - Non-Commercial Use Permitted

Originally published in:

SAM Research Report 1998-09 


\title{
Nonreflecting Boundary Conditions For Electromagnetic Scattering
}

\author{
M.J. Grote
}

Research Report No. 98-09

September 1998

Seminar für Angewandte Mathematik

Eidgenössische Technische Hochschule

CH-8092 Zürich

Switzerland 


\title{
Nonreflecting Boundary Conditions For Electromagnetic Scattering
}

\author{
M.J. Grote \\ Seminar für Angewandte Mathematik \\ Eidgenössische Technische Hochschule \\ CH-8092 Zürich \\ Switzerland
}

Research Report No. 98-09 September 1998

\begin{abstract}
An exact nonreflecting boundary condition was derived previously for use with the time dependent Maxwell equations in three space dimensions ${ }^{1}$. Here it is shown how to combine that boundary condition with the variational formulation for use with the finite element method. The fundamental theory underlying the derivation of the exact boundary condition is reviewed. Numerical examples with the finite-difference timedomain method are presented which demonstrate the high accuracy and confirm the expected rate of convergence of the numerical method.
\end{abstract}

Keywords: Maxwell's equations, electromagnetic scattering, nonreflecting boundary conditions, absorbing boundary conditions, finite element method, finite difference method

AMS Subject Classification: 65M06, 65M60, 78-08, 78A40 


\section{Introduction}

We wish to calculate numerically the time dependent electric field $\boldsymbol{E}(\boldsymbol{x}, t)$ and magnetic field $\boldsymbol{H}(\boldsymbol{x}, t)$ scattered from a bounded scattering region in three-dimensional space. In this region, there may be one or more scatterers and the equations for $\boldsymbol{E}$ and $\boldsymbol{H}$ may have variable coefficients and nonlinear terms. As usual, we surround the scattering region by an artificial boundary $\mathcal{B}$ and confine the computation to the region $\Omega$ bounded by $\mathcal{B}$. Then, to formulate the problem for $\boldsymbol{E}$ and $\boldsymbol{H}$ in $\Omega$, we make them satisfy a boundary condition on $\mathcal{B}$. The boundary conditions commonly imposed produce spurious reflections from $\mathcal{B}$. To avoid this spurious reflection we have devised an exact nonreflecting boundary condition ${ }^{1}$. It is the extension to Maxwell's equations of the exact nonreflecting boundary condition which we have derived for the scalar wave equation ${ }^{2,3}$. In doing so, we chose $\mathcal{B}$ to be a sphere of radius $R$, and we assumed that $\boldsymbol{E}$ and $\boldsymbol{H}$ satisfied the homogeneous Maxwell equations outside $\mathcal{B}$. The boundary condition is local in time and nonlocal on $\mathcal{B}$, and it does not involve high-order derivatives on $\mathcal{B}$.

Usually various approximate boundary conditions are used, which are local differential operators on $\mathcal{B}$. Examples are the Mur $^{4}$ and the Peterson ${ }^{5}$ conditions, which are the generalizations to Maxwell's equations of the absorbing boundary conditions derived for the scalar wave equation by Engquist and Majda ${ }^{6}$ and by Bayliss and Turkel ${ }^{7}$. Higdon ${ }^{8}$ showed that any local boundary condition involving a differential operator eliminates spurious reflections at certain angles of incidence but not at others. Recently Ramahi ${ }^{9}$ extended this approach by combining two full numerical solutions, each with a different (complementary) boundary operator. Although most approximate boundary conditions perform well at nearly normal incidence, their performance degrades rapidly as grazing incidence is approached. In complex situations the scattered waves arrive at the artificial boundary from all interior angles and at all frequencies, so these methods then yield some spurious reflection. Moreover, these errors tend to accumulate with time and prevent accurate long-time integration.

A different approach to eliminate reflection has been to append an artificial transition layer (PML) outside $\mathcal{B}$, which is supposed to absorb outgoing waves ${ }^{10,11,12}$. To minimize the amount of reflection and to achieve very high accuracy, it is often necessary to use a thick absorbing layer, which is expensive in computer storage and execution time. Recently a comparison of the PML technique ${ }^{10}$ with the nonreflecting boundary conditions ${ }^{1}$ was performed by Kantartzis et al. ${ }^{13}$.

An exact nonreflecting boundary condition for the wave equation was proposed by Ting and Miksis ${ }^{14}$. It is based on a Kirchhoff integral representation of the solution on $\mathcal{B}$ and requires storing the solution at a surface inside $\mathcal{B}$ for the length of time it takes a wave to propagate across $\Omega$. This approach was generalized to Maxwell's equations by De Moerloose and De Zutter ${ }^{15}$. To update the solution value at any point on the two-dimensional artificial boundary $\mathcal{B}$ requires a two-dimensional convolution in time and space. Therefore using this boundary condition may be more expensive than using the numerical scheme itself inside $\Omega$.

It is to avoid the various difficulties mentioned above that we have derived this new exact nonreflecting boundary condition ${ }^{1}$ for the special case when $\mathcal{B}$ is a sphere. In section 3 we review the basic theory underlying the derivation of the exact boundary condition in a special simple situation. Next, we show in section 4 that our boundary condition fits easily 
into the variational formulation of Maxwell's equations. Hence it is also well-suited for use with the finite element method. In section 5 we show how to combine it with the popular finite-difference time-domain method (FDTD) by Yee ${ }^{16}$. Finally in section 6, we present numerical results which confirm the expected high accuracy of our boundary condition.

\section{Formulation}

We consider time-dependent scattering from a bounded scattering region in three-dimensional space. We surround this region by a sphere $\mathcal{B}$ of radius $R$. In $\mathcal{B}^{\text {ext }}$, the region outside $\mathcal{B}$, we assume that the electric field $\boldsymbol{E}$ and the magnetic field $\boldsymbol{H}$ satisfy Maxwell's equations

$$
\varepsilon \frac{\partial \boldsymbol{E}}{\partial t}=\nabla \times \boldsymbol{H}, \quad \mu \frac{\partial \boldsymbol{H}}{\partial t}=-\nabla \times E .
$$

Here $\varepsilon$ and $\mu$ are the constant electric permittivity and the constant magnetic permeability, respectively.

We suppose that the domain $\Omega$ within $\mathcal{B}$ is bounded internally by a smooth surface $\Gamma$. In $\Omega$, we consider the following simple model problem:

$$
\begin{aligned}
\varepsilon \frac{\partial \boldsymbol{E}}{\partial t} & =\nabla \times \boldsymbol{H}-\boldsymbol{J}, \\
\mu \frac{\partial \boldsymbol{H}}{\partial t} & =-\nabla \times E, \quad \text { in } \Omega \times(0, T),
\end{aligned}
$$

where $\varepsilon$ and $\mu$ are known functions of $\boldsymbol{x}$ and $\boldsymbol{J}$ is a function of space and time giving the current density. At $t=0$ we specify the initial data

$$
\boldsymbol{E}(\boldsymbol{x}, 0)=\boldsymbol{E}_{0}(\boldsymbol{x}), \quad \boldsymbol{H}(\boldsymbol{x}, 0)=\boldsymbol{H}_{0}(\boldsymbol{x}), \quad \boldsymbol{x} \in \Omega,
$$

where $\boldsymbol{E}_{0}$ and $\boldsymbol{H}_{0}$ are given functions. On the internal physical boundary $\Gamma$ we assume the boundary condition

$$
\hat{\boldsymbol{n}} \times \boldsymbol{E}=\gamma, \quad \text { in } \Gamma \times(0, T),
$$

where $\gamma$ is a specified function and $\hat{\boldsymbol{n}}$ is the unit outward normal. On the exterior artificial boundary $\mathcal{B}$, we impose the boundary condition

$$
\begin{aligned}
\hat{\boldsymbol{r}} \times \nabla \times \boldsymbol{E}-\frac{1}{c} \frac{\partial \boldsymbol{E}^{\mathrm{tan}}}{\partial t} & =\frac{1}{R} \sum_{n \geq 1} \sum_{|m| \leq n} \boldsymbol{d}_{n} \cdot \boldsymbol{\psi}_{n m}^{E}(t) \boldsymbol{V}_{n m} \\
& -\sqrt{\frac{\mu}{\varepsilon}} \frac{1}{R} \sum_{n \geq 1} \sum_{|m| \leq n} \boldsymbol{d}_{n} \cdot \boldsymbol{\psi}_{n m}^{H}(t) \boldsymbol{U}_{n m}, \quad r=R .
\end{aligned}
$$

The vector functions $\boldsymbol{\psi}_{n m}^{E}(t)$ and $\boldsymbol{\psi}_{n m}^{H}(t)$ in (2.6) satisfy the ordinary differential equations

$$
\begin{array}{ll}
\frac{1}{c} \frac{d}{d t} \boldsymbol{\psi}_{n m}^{E}(t)=\boldsymbol{A}_{n} \boldsymbol{\psi}_{n m}^{E}(t)+\left(\left.\boldsymbol{E}^{\mathrm{tan}}\right|_{r=R}, \boldsymbol{V}_{n m}\right) \boldsymbol{e}_{n}, & \boldsymbol{\psi}_{n m}^{E}(0)=0 \\
\frac{1}{c} \frac{d}{d t} \boldsymbol{\psi}_{n m}^{H}(t)=\boldsymbol{A}_{n} \boldsymbol{\psi}_{n m}^{H}(t)+\left(\left.\boldsymbol{H}^{\mathrm{tan}}\right|_{r=R}, \boldsymbol{V}_{n m}\right) \boldsymbol{e}_{n}, & \boldsymbol{\psi}_{n m}^{H}(0)=0
\end{array}
$$


Here $c=1 / \sqrt{\varepsilon \mu}$ is the speed of propagation and $\boldsymbol{U}_{n m}$ and $\boldsymbol{V}_{n m}$ denote the $n m$-th vector spherical harmonics

$$
\begin{aligned}
& \boldsymbol{U}_{n m}(\vartheta, \phi)=\frac{r \nabla Y_{n m}}{\sqrt{n(n+1)}}=\frac{1}{\sqrt{n(n+1)}}\left[\frac{\partial Y_{n m}}{\partial \vartheta} \hat{\boldsymbol{\vartheta}}+\frac{1}{\sin \vartheta} \frac{\partial Y_{n m}}{\partial \phi} \hat{\boldsymbol{\phi}}\right], \\
& \boldsymbol{V}_{n m}(\vartheta, \phi)=\hat{\boldsymbol{r}} \times \boldsymbol{U}_{n m}=\frac{1}{\sqrt{n(n+1)}}\left[\frac{-1}{\sin \vartheta} \frac{\partial Y_{n m}}{\partial \phi} \hat{\boldsymbol{\vartheta}}+\frac{\partial Y_{n m}}{\partial \vartheta} \hat{\boldsymbol{\phi}}\right] .
\end{aligned}
$$

They form an orthonormal basis for the space of tangential $L_{2}$ fields on the unit sphere with respect to the $L_{2}$ inner product ${ }^{17}$. In (2.9) and (2.10) we have introduced the polar coordinates $r, \vartheta, \phi$ and the unit vectors $\hat{\boldsymbol{r}}, \hat{\boldsymbol{\vartheta}}, \hat{\boldsymbol{\phi}}$. In addition, we let $Y_{n m}$ denote the $n m$-th spherical harmonic normalized over the unit sphere

$$
Y_{n m}(\vartheta, \phi)=\sqrt{\frac{(2 n+1)(n-|m|) !}{4 \pi(n+|m|) !}} P_{n}^{|m|}(\cos \vartheta) e^{i m \phi}, \quad n \geq 0,|m| \leq n .
$$

If the problem considered is real, one can use real instead of complex spherical harmonics. In that case everything remains the same, but the normalization constant in (2.11) will change.

Equation (2.6) is the exact nonreflecting boundary condition which was derived by Grote and Keller ${ }^{1}$. It involves the $n$-component vector functions $\boldsymbol{\psi}_{n m}^{H}(t)$ and $\boldsymbol{\psi}_{n m}^{E}(t)$, which are solutions of the linear first-order ordinary differential equations (2.7) and (2.8). In (2.6) the constant $n$-component vectors $\boldsymbol{d}_{n}=\left\{d_{n}^{j}\right\}$ are defined by

$$
d_{n}^{j}=\frac{n(n+1) j}{2 R^{j}}, \quad j=1, \ldots, n .
$$

The constant $n \times n$ matrices $\boldsymbol{A}_{n}=\left\{A_{n}^{i j}\right\}$ in (2.7) and (2.8) are defined by

$$
A_{n}^{i j}= \begin{cases}-n(n+1) /\left(2 R^{j}\right) & \text { if } i=1 \\ (n+i)(n+1-i) /(2 i) & \text { if } i=j+1 \\ 0 & \text { otherwise }\end{cases}
$$

and $\boldsymbol{e}_{n}=\left\{e_{n}^{j}\right\}$ denotes the constant $n$-component unit vector

$$
\boldsymbol{e}_{n}=[1,0, \ldots, 0]^{\top} \text {. }
$$

If we set the right side of (2.6) equal to zero we obtain the first-order Peterson condition ${ }^{5}$, the time dependent counterpart of the Silver-Müller radiation condition for the time-harmonic case $^{17}$.

\section{Derivation of the exact nonreflecting boundary condition}

\subsection{The multipole decomposition}

To solve (2.1) in $\mathcal{B}^{\text {ext }}$, we decompose the electromagnetic field into transverse electric (TE) and transverse magnetic (TM) fields. The electric component of the TE multipole field of 
order $(n, m)$ is given by

$$
\boldsymbol{E}_{n m}^{T E}(r, \vartheta, \phi, t)=f_{n m}(r, t) \boldsymbol{V}_{n m}(\vartheta, \phi)
$$

where $f_{n m}$ satisfies

$$
L_{n}\left[f_{n m}\right] \equiv\left(\frac{1}{c^{2}} \frac{\partial^{2}}{\partial t^{2}}-\frac{\partial^{2}}{\partial r^{2}}-\frac{2}{r} \frac{\partial}{\partial r}+\frac{n(n+1)}{r^{2}}\right) f_{n m}=0 .
$$

The magnetic component of the TM multipole field of order $(n, m)$ is given by

$$
\boldsymbol{H}_{n m}^{T M}(r, \vartheta, \phi, t)=g_{n m}(r, t) \boldsymbol{V}_{n m}(\vartheta, \phi),
$$

where $L_{n}\left[g_{n m}\right]=0$.

The TE and TM solutions form a complete set of solutions of Maxwell's equations in a source-free region ${ }^{18}$ (p. 746). Therefore the general electric and magnetic multipole field of order $(n, m)$ are obtained by combining (3.1) with the electric field associated with (3.3), and combining (3.3) with the magnetic field associated with (3.1), respectively:

$$
\begin{aligned}
& \boldsymbol{E}_{n m}(r, \vartheta, \phi, t)=f_{n m}(r, t) \boldsymbol{V}_{n m}+\frac{1}{\varepsilon} \nabla \times\left[\boldsymbol{V}_{n m} \int_{0}^{t} g_{n m}(r, s) d s\right], \\
& \boldsymbol{H}_{n m}(r, \vartheta, \phi, t)=-\frac{1}{\mu} \nabla \times\left[\boldsymbol{V}_{n m} \int_{0}^{t} f_{n m}(r, s) d s\right]+g_{n m}(r, t) \boldsymbol{V}_{n m} .
\end{aligned}
$$

In $\mathcal{B}^{\text {ext }}$, the total electromagnetic field is a superposition of multipole fields:

$$
\boldsymbol{E}=\sum_{n \geq 1} \sum_{|m| \leq n} \boldsymbol{E}_{n m}, \quad \boldsymbol{H}=\sum_{n \geq 1} \sum_{|m| \leq n} \boldsymbol{H}_{n m}
$$

The vector spherical harmonics satisfy the following useful equations for any $f \in C^{1}$ :

$$
\begin{aligned}
& \nabla \times\left(f(r) \boldsymbol{V}_{n m}\right)=-\frac{\sqrt{n(n+1)} f(r)}{r} Y_{n m} \hat{\boldsymbol{r}}-\frac{1}{r} \frac{\partial(r f(r))}{\partial r} \boldsymbol{U}_{n m}, \\
& \hat{\boldsymbol{r}} \times \nabla \times\left(f(r) \boldsymbol{V}_{n m}\right)=-\frac{1}{r} \frac{\partial(r f(r))}{\partial r} \boldsymbol{V}_{n m} .
\end{aligned}
$$

By using (3.7) we see that the terms in (3.4) and (3.5) that involve $\nabla \times\left(\boldsymbol{V}_{n m} \ldots\right)$ are orthogonal to $\boldsymbol{V}_{n m}$. Thus from (3.6) we conclude that

$$
f_{n m}=\left(\boldsymbol{E}, \boldsymbol{V}_{n m}\right), \quad g_{n m}=\left(\boldsymbol{H}, \boldsymbol{V}_{n m}\right) .
$$

The inner products involve integration with respect to $\vartheta$ and $\phi$ on the sphere of radius $r$ and apply only to the tangential components of $\boldsymbol{E}$ and $\boldsymbol{H}$. 


\subsection{The case $n=1$}

Previously we showed how the exact nonreflecting boundary conditions for the scalar wave equation $^{2,3}$ could be used to derive the corresponding nonreflecting boundary condition for Maxwell's equations ${ }^{1}$. We shall not repeat this analysis in its full generality, but instead restrict ourselves to the special case $n=1$. This greatly simplifies the algebra and enables us to concentrate on the main ideas underlying the derivation of the boundary condition. Thus, we consider an electromagnetic field, which consists only of a first order multipole (dipole) with $n=1$ and $m=-1, m=0$, or $m=1$. Hence $\boldsymbol{E}$ is given by (3.4) with $n=1$ :

$$
\boldsymbol{E}(r, \vartheta, \phi, t)=f(r, t) \boldsymbol{V}_{1 m}+\frac{1}{\varepsilon} \nabla \times\left[\boldsymbol{V}_{1 m} \int_{0}^{t} g(r, s) d s\right], \quad r \geq R .
$$

Here $f$ and $g$ - we omit the indices $(n, m)$ on $f$ and $g$ - both satisfy (3.2) with $n=1$.

First we apply $\hat{\boldsymbol{r}} \times \nabla \times$ to (3.10) and evaluate $\hat{\boldsymbol{r}} \times \nabla \times\left[f \boldsymbol{V}_{1 m}\right]$ by using (3.8). To compute the last term in the resulting expression, we recall that $\boldsymbol{V}_{1 m} \int_{0}^{t} g$ is a solution of the vector wave equation

$$
\frac{1}{c^{2}} \frac{\partial^{2} \boldsymbol{H}}{\partial t^{2}}+\nabla \times \nabla \times \boldsymbol{H}=0, \quad r \geq R .
$$

As a consequence,

$$
\hat{\boldsymbol{r}} \times \nabla \times \nabla \times\left[\boldsymbol{V}_{1 m} \int_{0}^{t} g(r, s) d s\right]=-\hat{\boldsymbol{r}} \times\left[\frac{1}{c^{2}} \frac{\partial g}{\partial t} \boldsymbol{V}_{1 m}\right]=\frac{1}{c^{2}} \frac{\partial g}{\partial t} \boldsymbol{U}_{1 m}
$$

Since $\varepsilon^{-1} c^{-2}=(\mu / \varepsilon)^{1 / 2} c^{-1}$, we get

$$
\hat{\boldsymbol{r}} \times \nabla \times \boldsymbol{E}=-\frac{1}{r} \frac{\partial(r f)}{\partial r} \boldsymbol{V}_{1 m}+\sqrt{\frac{\mu}{\varepsilon}} \frac{1}{c} \frac{\partial g}{\partial t} \boldsymbol{U}_{1 m} .
$$

Next we differentiate (3.10) with respect to $t$ and simplify the result using (3.7). The tangential components of the resulting expression yield

$$
\frac{1}{c} \frac{\partial \boldsymbol{E}^{\mathrm{tan}}}{\partial t}=\frac{1}{c} \frac{\partial f}{\partial t} \boldsymbol{V}_{1 m}-\sqrt{\frac{\mu}{\varepsilon}} \frac{1}{r} \frac{\partial(r g)}{\partial r} \boldsymbol{U}_{1 m} .
$$

Now we subtract (3.14) from (3.13) to get

$$
\hat{\boldsymbol{r}} \times \nabla \times \boldsymbol{E}-\frac{1}{c} \frac{\partial \boldsymbol{E}^{\tan }}{\partial t}=-\frac{1}{r}\left(\frac{\partial}{\partial r}+\frac{1}{c} \frac{\partial}{\partial t}\right)[r f] \boldsymbol{V}_{1 m}+\sqrt{\frac{\mu}{\varepsilon}} \frac{1}{r}\left(\frac{\partial}{\partial r}+\frac{1}{c} \frac{\partial}{\partial t}\right)[r g] \boldsymbol{U}_{1 m}
$$

Equation (3.15) cannot yet be used as a boundary condition because it involves unknown radial and time derivatives of $f$ and $g$.

We need to express these derivatives in terms of known quantities, which do not involve the past of the solution. To do so, we first note that both $f$ and $g$ satisfy the differential equation (3.2) with $n=1$, and that they both vanish at $t=0$ for $r \geq R$. Next, we recall the following result: let $v(r, t)$ be a solution of the one dimensional wave equation $v_{t t}-c^{2} v_{r r}=0$. Then $u(r, t)$, defined by

$$
u=\frac{\partial}{\partial r} \frac{v}{r}
$$


satisfies $L_{1}[u]=0$, with $L_{1}$ as in (3.2) with $n=1$. As $L_{1}[f]=0$, we invert the above result with $u$ replaced by $f$. Let

$$
v(r, t)=r \int_{r}^{\infty} f(s, t) d s
$$

then $v_{t t}-c^{2} v_{r r}=0$. Since $f$ and $\partial_{t} f$ both vanish at $t=0$ for $r \geq R$, so do $v$ and $\partial_{t} v$ for $r \geq R$. Therefore $v(r, t)$ is a purely outgoing solution of the one dimensional wave equation and satisifies

$$
\left(\frac{\partial}{\partial r}+\frac{1}{c} \frac{\partial}{\partial t}\right) v(r, t)=0, \quad r \geq R .
$$

Although (3.18) is an exact boundary condition on $v$, and hence implicitly on $f$, we cannot use it as such and need to recast it into a form which involves only $f$. To do so, we differentiate (3.16) with respect to $r$ and use (3.18) to obtain

$$
\frac{1}{c} \frac{\partial}{\partial t} v=-\frac{\partial}{\partial r} v=r f-\int_{r}^{\infty} f(s, t) d s=r f-\frac{v}{r} .
$$

Next, we apply $\partial_{r}+c^{-1} \partial_{t}$ to (3.19), simplify the result with (3.18), and set $r=R$ to obtain

$$
\left(\frac{\partial}{\partial r}+\frac{1}{c} \frac{\partial}{\partial t}\right) r f(r, t)=-\frac{v(R, t)}{R^{2}}, \quad r=R .
$$

Equation (3.20) is an exact boundary condition precisely for the quantity we are interested in, but it still involves the a priori unknown quantity $v(R, t)$. To determine it we set $r=R$ in (3.19) which yields an ordinary differential equation for $v(R, t)$. With $\boldsymbol{\psi}^{E}(t)=v(R, t) / R$, the boundary condition (3.20) together with (3.19) becomes

$$
\begin{aligned}
\left(\frac{\partial}{\partial r}+\frac{1}{c} \frac{\partial}{\partial t}\right)[r f] & =-\frac{\boldsymbol{\psi}^{E}}{R}, \quad r=R, \\
\frac{1}{c} \frac{d \boldsymbol{\psi}^{E}}{d t} & =-\frac{\boldsymbol{\psi}^{E}}{R}+\left(\left.\boldsymbol{E}\right|_{r=R}, \boldsymbol{V}_{1 m}\right), \quad \boldsymbol{\psi}^{E}(0)=0 .
\end{aligned}
$$

We recall that $\boldsymbol{\psi}^{E}(0)=0$ because $v(r, t)$ vanishes identically for $r \geq R$ at $t=0$. Similarly, we derive the exact boundary condition for $g(r, t)$ at $r=R$,

$$
\begin{aligned}
\left(\frac{\partial}{\partial r}+\frac{1}{c} \frac{\partial}{\partial t}\right)[r g] & =-\frac{\boldsymbol{\psi}^{H}}{R}, \quad r=R, \\
\frac{1}{c} \frac{d \boldsymbol{\psi}^{H}}{d t} & =-\frac{\boldsymbol{\psi}^{H}}{R}+\left(\left.\boldsymbol{H}\right|_{r=R}, \boldsymbol{V}_{1 m}\right), \quad \boldsymbol{\psi}^{H}(0)=0 .
\end{aligned}
$$

Finally, we use (3.21) and (3.23) to replace the terms in $f$ and $g$ that appear in (3.15). This yields the exact nonreflecting boundary condition when $E$ consists only of a first order multipole, $n=1$ and $|m| \leq 1$,

$$
\hat{\boldsymbol{r}} \times \nabla \times \boldsymbol{E}-\frac{1}{c} \frac{\partial \boldsymbol{E}^{\tan }}{\partial t}=\frac{\boldsymbol{\psi}^{E}(t)}{R^{2}} \boldsymbol{V}_{1 m}-\sqrt{\frac{\mu}{\varepsilon}} \frac{\boldsymbol{\psi}^{H}(t)}{R^{2}} \boldsymbol{U}_{1 m}, \quad r=R .
$$

Here $\boldsymbol{\psi}^{E}(t)$ and $\boldsymbol{\psi}^{H}(t)$ are determined by the linear first order ordinary differential equations in (3.22) and (3.24). 


\subsection{The full electromagnetic field}

The procedure presented in the previous section for the special case $n=1$ can be extended to the general case for all $n \geq 1$. Then each multipole $(n, m)$ leads to functions $\boldsymbol{\psi}_{n m}^{E}(t)$ and $\boldsymbol{\psi}_{n m}^{H}(t)$, which satisfy linear first-order systems of ordinary differential equations. Finally, we obtain the boundary condition at $r=R$ by summing over $n$ and $m$. This yields the boundary condition for $\boldsymbol{E}$ in (2.6). The boundary condition for $\boldsymbol{H}$ is given by

$$
\begin{aligned}
\hat{\boldsymbol{r}} \times \nabla \times \boldsymbol{H}-\frac{1}{c} \frac{\partial \boldsymbol{H}^{\mathrm{tan}}}{\partial t} & =\sqrt{\frac{\varepsilon}{\mu}} \frac{1}{R} \sum_{n \geq 1} \sum_{|m| \leq n} \boldsymbol{d}_{n} \cdot \boldsymbol{\psi}_{n m}^{E}(t) \boldsymbol{U}_{n m} \\
& +\frac{1}{R} \sum_{n \geq 1} \sum_{|m| \leq n} \boldsymbol{d}_{n} \cdot \boldsymbol{\psi}_{n m}^{H}(t) \boldsymbol{V}_{n m}, \quad r=R .
\end{aligned}
$$

Here $\boldsymbol{d}_{n}$ is defined by (2.12) and the functions $\boldsymbol{\psi}_{n m}^{E}(t)$ and $\boldsymbol{\psi}_{n m}^{H}(t)$ are defined by (2.7) and (2.8).

Each inner product in (2.7) and (2.8) involves two, not three, scalar inner products since $\boldsymbol{V}_{n m}$ is purely tangential. Equations (3.26) and (2.6) are the boundary conditions which we sought. They are exact and ensure that no spurious reflections occur at $\mathcal{B}$. They involve only first-order derivatives and can be incorporated easily into numerical methods.

The functions $f_{n m}$ and $g_{n m}$ satisfy the scalar wave equation and determine the electromagnetic field uniquely outside $\mathcal{B}$. Previously it was shown that for smooth solutions of the scalar wave equation, imposing the exact boundary condition at $\mathcal{B}$ guarantees that the solution in $\Omega$ coincides with the restriction to $\Omega$ of the solution to the Cauchy problem in the unbounded domain ${ }^{3}$. Therefore for smooth data, the solution to the initial boundary value problem inside $\Omega$ with (3.26) or (2.6) imposed at $\mathcal{B}$, is unique and coincides with the restriction to $\Omega$ of the electromagnetic field in the unbounded domain. It is also well-posed with respect to perturbations in the initial conditions.

In practice, the infinite sums in (3.26) and (2.6) must be truncated at some finite value $N$. For the modes $n>N$, the truncated boundary condition for $\boldsymbol{E}$ reduces to

$$
\hat{\boldsymbol{r}} \times \nabla \times \boldsymbol{E}-\frac{1}{c} \frac{\partial \boldsymbol{E}^{\tan }}{\partial t}=0 .
$$

This is the time-dependent counterpart of the first-order approximate boundary condition derived by Peterson ${ }^{5}$, which annihilates the leading term in the large distance expansion of the electromagnetic field ${ }^{19}$.

The truncation at $N$ introduces an error $O\left(R^{-3}\right)$ in modes with $n>N$. To further reduce the error on the modes $n>N$, without affecting the modes $n \leq N$, one can simply apply higher order boundary conditions to both sides of (3.26) and (2.6). The resulting boundary conditions are still exact, but when truncated at $n=N$ the error on the higher modes is smaller. Then the vector functions $\boldsymbol{\psi}_{n m}^{E}(t)$ and $\boldsymbol{\psi}_{n m}^{H}(t)$ satisfy the same ordinary differential equations (2.7) and (2.8). Only the constant vectors $\boldsymbol{d}_{n}$ in (2.12) need to be recalculated. This procedure is illustrated in Reference 1 for the second-order Peterson condition:

$$
\left\{\hat{\boldsymbol{r}} \times(\nabla \times)-\frac{1}{c} \frac{\partial}{\partial t}-\frac{2}{r}\right\}\left\{\hat{\boldsymbol{r}} \times \nabla \times \boldsymbol{E}-\frac{1}{c} \frac{\partial \boldsymbol{E}^{\mathrm{tan}}}{\partial t}\right\}=0, \quad r=R .
$$


The error in (3.28) is $O\left(R^{-5}\right)$, which is smaller than the error $O\left(R^{-3}\right)$ in $(3.27)$ for $R>1$.

These boundary conditions do not require saving past values of $\boldsymbol{E}$ or $\boldsymbol{H}$. Instead they involve the two functions $\boldsymbol{\psi}_{n m}^{E}(t)$ and $\boldsymbol{\psi}_{n m}^{H}(t)$. The amount of memory needed to store them, about $(4 / 3) N^{3}$ scalar values, is negligible when compared to the storage required for $\boldsymbol{E}$ and $\boldsymbol{H}$. Most of the extra work involved in applying the boundary condition results from computing the inner products of $\boldsymbol{E}$ and $\boldsymbol{H}$ with $\boldsymbol{V}_{n m}$ in (2.7) and (2.8), and from computing the right-hand sides of (3.26) and (2.6).

To compute the Fourier components in (3.26) or (2.6), it is not necessary to compute $O\left(N^{2}\right)$ inner products over the entire sphere. Indeed, since the vector spherical harmonics $\boldsymbol{U}_{n m}$ and $\boldsymbol{V}_{n m}$ separate in $\theta$ and $\phi$, it is sufficient to compute $O(N)$ inner products with $\cos (m \phi)$ and $\sin (m \phi)$ over the sphere, and then to compute $O\left(N^{2}\right)$ one-dimensional inner products in $\theta$ over $[0, \pi]$. The same method can be used to calculate the sums over $n$ and $m$ on the right of (3.26) and (2.6). In all our computations we have found $N \leq 25$ to be sufficient. If very large values of $N$ were needed, the work and storage required could be reduced by an order of magnitude by combining a fast spherical harmonic transform over the sphere ${ }^{20}$ with the recent work of Alpert, Greengard, and Hagstrom ${ }^{21}$ on the approximate solution of (2.7) and (2.8).

\section{The finite element formulation}

We shall now derive the finite element formulation for the problem $(2.1)-(2.6)$ in the computational domain $\Omega$. We let

$$
H(\operatorname{curl} ; \Omega)=\left\{\boldsymbol{w} \in\left(L^{2}(\Omega)\right)^{3} \mid \nabla \times \boldsymbol{w} \in\left(L^{2}(\Omega)\right)^{3}\right\},
$$

and denote by $(., .)_{\Omega},(., .)_{\Gamma}$, and $(., .)_{\mathcal{B}}$ the inner products over $\Omega, \Gamma$, and $\mathcal{B}$. To derive the weak or variational formulation of $(2.1)-(2.6)$ we multiply the first equation in (2.1) by a test function $\Theta(\boldsymbol{x})$ and the second equation of (2.1) by a test function $\boldsymbol{\Phi}(\boldsymbol{x})$. By integrating the resulting expressions over $\Omega$ we obtain

$$
\begin{aligned}
\left(\varepsilon \partial_{t} \boldsymbol{E}, \boldsymbol{\Theta}\right)_{\Omega} & =(\nabla \times \boldsymbol{H}, \boldsymbol{\Theta})_{\Omega}-(J, \boldsymbol{\Theta})_{\Omega}, \\
\left(\mu \partial_{t} \boldsymbol{H}, \boldsymbol{\Phi}\right)_{\Omega} & =-(\nabla \times \boldsymbol{E}, \boldsymbol{\Phi})_{\Omega} .
\end{aligned}
$$

Then we use integration by parts in (4.3) to get

$$
\left(\mu \partial_{t} \boldsymbol{H}, \boldsymbol{\Phi}\right)_{\Omega}=(\boldsymbol{E}, \nabla \times \boldsymbol{\Phi})_{\Omega}-(\hat{\boldsymbol{n}} \times \boldsymbol{E}, \boldsymbol{\Phi})_{\Gamma}-(\hat{\boldsymbol{r}} \times \boldsymbol{E}, \boldsymbol{\Phi})_{\mathcal{B}} .
$$

The ECHL method (E constant, H linear) of Lee and Madsen ${ }^{22}$ is based on this procedure. For alternative variational formulations see $\mathrm{Monk}^{23}$. In (4.4) we must specify $\hat{\boldsymbol{n}} \times \boldsymbol{E}$ on $\Gamma$ and $\hat{\boldsymbol{r}} \times \boldsymbol{E}$ on $\mathcal{B}$. The former is given immediately by (2.5), but the latter cannot be determined directly from (2.6).

We shall now reformulate the nonreflecting boundary condition (2.6) to determine the tangential components $\hat{\boldsymbol{r}} \times \boldsymbol{E}$ at $\mathcal{B}$ required in (4.4). We begin by introducing

$$
\Psi_{n m}^{E}(t)=\int_{0}^{t} \boldsymbol{\psi}_{n m}^{E}(s) d s, \quad \Psi_{n m}^{H}(t)=\int_{0}^{t} \boldsymbol{\psi}_{n m}^{H}(s) d s .
$$


Therefore, $\Psi_{n m}^{E}$ and $\Psi_{n m}^{H}$ satisfy the same ordinary differential equations (2.7)-(2.8) as $\boldsymbol{\psi}_{n m}^{E}$ and $\boldsymbol{\psi}_{n m}^{H}$, but with $f_{n m}$ and $g_{n m}$ replaced by their time integrals. To determine these time integrals we first compute $\hat{\boldsymbol{r}} \cdot \partial_{t} \boldsymbol{E}_{n m}$ in (3.4) and use (3.7). This yields

$$
\hat{\boldsymbol{r}} \cdot \frac{\partial \boldsymbol{E}_{n m}}{\partial t}=-\frac{\sqrt{n(n+1)}}{\varepsilon R} g_{n m} Y_{n m}, \quad r=R .
$$

By taking the inner product of (4.6) with $Y_{n m}$ we get

$$
g_{n m}=-\frac{\varepsilon R}{\sqrt{n(n+1)}}\left(\hat{\boldsymbol{r}} \cdot \frac{\partial \boldsymbol{E}}{\partial t}, Y_{n m}\right)_{\mathcal{B}}, \quad r=R .
$$

Similarly,

$$
f_{n m}=\frac{\mu R}{\sqrt{n(n+1)}}\left(\hat{\boldsymbol{r}} \cdot \frac{\partial \boldsymbol{H}}{\partial t}, Y_{n m}\right)_{\mathcal{B}}, \quad r=R .
$$

By integrating (4.7) and (4.8) in time, we conclude that $\boldsymbol{\Psi}_{n m}^{E}$ is the solution of

$$
\frac{1}{c} \frac{d}{d t} \boldsymbol{\Psi}_{n m}^{E}(t)=\boldsymbol{A}_{n} \boldsymbol{\Psi}_{n m}^{E}(t)+\frac{\mu R}{\sqrt{n(n+1)}}\left(\left.\hat{\boldsymbol{r}} \cdot \boldsymbol{H}\right|_{r=R}, Y_{n m}\right)_{\mathcal{B}} \boldsymbol{e}_{n}, \quad \boldsymbol{\Psi}_{n m}^{E}(0)=0
$$

and that $\Psi_{n m}^{H}$ is the solution of

$$
\frac{1}{c} \frac{d}{d t} \Psi_{n m}^{H}(t)=\boldsymbol{A}_{n} \Psi_{n m}^{H}(t)-\frac{\varepsilon R}{\sqrt{n(n+1)}}\left(\left.\hat{\boldsymbol{r}} \cdot \boldsymbol{E}\right|_{r=R}, Y_{n m}\right)_{\mathcal{B}} \boldsymbol{e}_{n}, \quad \boldsymbol{\Psi}_{n m}^{H}(0)=0
$$

Next, we integrate $(2.6)$ with respect to time. The right side remains the same, with $\boldsymbol{\psi}_{n m}^{E}$ and $\boldsymbol{\psi}_{n m}^{H}$ replaced by $\Psi_{n m}^{E}$ and $\boldsymbol{\Psi}_{n m}^{H}$. The left side can be reformulated easily using (2.1), which leads to the alternative formulation of the nonreflecting boundary conditions at $r=R$ :

$$
\varepsilon \hat{\boldsymbol{r}} \times \boldsymbol{E}-\frac{1}{c} \boldsymbol{H}^{\mathrm{tan}}=\frac{1}{R} \sum_{n \geq 1} \sum_{|m| \leq n}\left\{\sqrt{\frac{\varepsilon}{\mu}} \boldsymbol{d}_{n} \cdot \Psi_{n m}^{E}(t) \boldsymbol{U}_{n m}+\boldsymbol{d}_{n} \cdot \boldsymbol{\Psi}_{n m}^{H}(t) \boldsymbol{V}_{n m}\right\} .
$$

Equation (4.11) does not involve any derivatives of $\boldsymbol{E}$ or $\boldsymbol{H}$. It is quite remarkable that the two scalar quantities $\hat{\boldsymbol{r}} \cdot \boldsymbol{E}$ and $\hat{\boldsymbol{r}} \cdot \boldsymbol{H}$ in (4.9) and (4.10) suffice to impose the nonreflecting boundary condition.

We now proceed with the derivation of the finite element formulation and use (4.11) to replace $\hat{\boldsymbol{r}} \times \boldsymbol{E}$ at $\mathcal{B}$ in (4.4). This leads to the weak form of the problem, which can be stated as follows:

Find $\boldsymbol{E}(t) \in\left(L^{2}(\Omega)\right)^{3}$ and $\boldsymbol{H}(t) \in H(\operatorname{curl} ; \Omega)$ such that for all $\boldsymbol{\Phi} \in H(\operatorname{curl} ; \Omega)$ and for all $\boldsymbol{\Theta} \in\left(L^{2}(\Omega)\right)^{3}$,

$$
\begin{aligned}
\left(\varepsilon \partial_{t} \boldsymbol{E}, \boldsymbol{\Theta}\right)_{\Omega} & =(\nabla \times \boldsymbol{H}, \boldsymbol{\Theta})_{\Omega}-(J, \boldsymbol{\Theta})_{\Omega} \\
\left(\mu \partial_{t} \boldsymbol{H}, \boldsymbol{\Phi}\right)_{\Omega} & =(\boldsymbol{E}, \nabla \times \boldsymbol{\Phi})_{\Omega}-(\gamma, \boldsymbol{\Phi})_{\Gamma}-\sqrt{\frac{\mu}{\varepsilon}}\left(\boldsymbol{H}^{\tan }, \boldsymbol{\Phi}\right)_{\mathcal{B}} \\
& -\frac{1}{R c} \sum_{n \geq 1} \sum_{|m| \leq n}\left\{\boldsymbol{d}_{n} \cdot \boldsymbol{\Psi}_{n m}^{E}\left(\boldsymbol{U}_{n m}, \boldsymbol{\Phi}\right)_{\mathcal{B}}+\sqrt{\frac{\mu}{\varepsilon}} \boldsymbol{d}_{n} \cdot \boldsymbol{\Psi}_{n m}^{H}\left(\boldsymbol{V}_{n m}, \boldsymbol{\Phi}\right)_{\mathcal{B}}\right\},
\end{aligned}
$$


together with the initial conditions (2.4). Here we have used the fact that $\varepsilon$ and $\mu$ are constant on $\mathcal{B}$. We note that with this formulation neither trial nor test functions need to satisfy any boundary conditions. The boundary conditions on $\boldsymbol{E}$ at $\Gamma$ and $\mathcal{B}$ are imposed naturally through the variational formulation.

The finite element method is obtained by approximating the weak form (4.12). To discretize in space we choose the finite-dimensional spaces $\mathcal{V}^{h} \subset\left(L^{2}(\Omega)\right)^{3}$ and $\mathcal{W}^{h} \subset H(\operatorname{curl} ; \Omega)$. Then we approximate the electric and the magnetic fields by

$$
\boldsymbol{E}^{h}(\boldsymbol{x}, t)=\sum_{i \in \eta_{E}} E_{i}(t) \boldsymbol{\Theta}_{i}(\boldsymbol{x}), \quad \boldsymbol{H}^{h}(\boldsymbol{x}, t)=\sum_{j \in \eta_{H}} H_{j}(t) \boldsymbol{\Phi}_{j}(\boldsymbol{x}),
$$

where $\eta_{E}$ and $\eta_{H}$ are the sets of indices representing the degrees of freedom for $\boldsymbol{E}^{h}$ and $\boldsymbol{H}^{h}$, respectively, and $\left\{\boldsymbol{\Theta}_{i}(\boldsymbol{x})\right\}$ and $\left\{\boldsymbol{\Phi}_{i}(\boldsymbol{x})\right\}$ are suitable basis functions. We denote by $\beta_{E} \subset \eta_{E}$ and $\beta_{H} \subset \eta_{H}$ the sets of indices representing the degrees of freedom for $\boldsymbol{E}^{h}$ and $\boldsymbol{H}^{h}$ located at the artificial boundary $\mathcal{B}$. In $(4.12)$ we truncate the sum over $n$ at some finite value $N$ and use (4.13) to obtain the finite element formulation of the problem:

Find $\left(\boldsymbol{E}^{h}(t), \boldsymbol{H}^{h}(t)\right) \in \mathcal{V}^{h} \times \mathcal{W}^{h}$ such that for all $\Theta_{k} \in \mathcal{V}^{h}$ and for all $\boldsymbol{\Phi}_{k} \in \mathcal{W}^{h}$

$$
\begin{aligned}
\left(\varepsilon \boldsymbol{\Theta}_{i}, \boldsymbol{\Theta}_{k}\right)_{\Omega} \frac{d E_{i}(t)}{d t} & =\left(\nabla \times \boldsymbol{\Phi}_{j}, \boldsymbol{\Theta}_{k}\right)_{\Omega} H_{j}(t)-\left(J, \boldsymbol{\Theta}_{k}\right)_{\Omega}, \\
\left(\mu \boldsymbol{\Phi}_{j}, \boldsymbol{\Phi}_{k}\right)_{\Omega} \frac{d H_{j}(t)}{d t} & =\left(\boldsymbol{\Theta}_{i}, \nabla \times \boldsymbol{\Phi}_{k}\right)_{\Omega} E_{i}(t)-\left(\gamma, \boldsymbol{\Phi}_{k}\right)_{\Gamma}-\sqrt{\frac{\mu}{\varepsilon}}\left(\boldsymbol{\Phi}_{j}^{\mathrm{tan}}, \boldsymbol{\Phi}_{k}\right)_{\mathcal{B}} H_{j}(t) \\
& -\frac{1}{R c} \sum_{n \geq 1} \sum_{|m| \leq n}\left\{\boldsymbol{d}_{n} \cdot \boldsymbol{\Psi}_{n m}^{E}\left(\boldsymbol{U}_{n m}, \boldsymbol{\Phi}_{k}\right)_{\mathcal{B}}+\sqrt{\frac{\mu}{\varepsilon}} \boldsymbol{d}_{n} \cdot \boldsymbol{\Psi}_{n m}^{H}\left(\boldsymbol{V}_{n m}, \boldsymbol{\Phi}_{k}\right)_{\mathcal{B}}\right\},
\end{aligned}
$$

with the initial conditions

$$
\begin{aligned}
& \left(\boldsymbol{\Theta}_{i}, \boldsymbol{\Theta}_{k}\right)_{\Omega} E_{i}(0)=\left(\boldsymbol{E}_{0}, \boldsymbol{\Theta}_{k}\right)_{\Omega}, \quad i, k \in \eta_{E}, \\
& \left(\boldsymbol{\Phi}_{j}, \boldsymbol{\Phi}_{k}\right)_{\Omega} H_{j}(0)=\left(\boldsymbol{H}_{0}, \boldsymbol{\Phi}_{k}\right)_{\Omega}, \quad j, k \in \eta_{H} .
\end{aligned}
$$

The auxilliary variables $\boldsymbol{\Psi}_{n m}^{E}(t)$ and $\boldsymbol{\Psi}_{n m}^{H}(t)$ satisfy

$$
\begin{aligned}
\frac{1}{c} \frac{d}{d t} \boldsymbol{\Psi}_{n m}^{E}(t) & =\boldsymbol{A}_{n} \boldsymbol{\Psi}_{n m}^{E}(t)+\frac{\mu R}{\sqrt{n(n+1)}} \sum_{i \in \beta_{H}} H_{i}(t)\left(\hat{\boldsymbol{r}} \cdot \boldsymbol{\Theta}_{i}, Y_{n m}\right)_{\mathcal{B}} \boldsymbol{e}_{n}, \\
\frac{1}{c} \frac{d}{d t} \boldsymbol{\Psi}_{n m}^{H}(t) & =\boldsymbol{A}_{n} \boldsymbol{\Psi}_{n m}^{H}(t)-\frac{\varepsilon R}{\sqrt{n(n+1)}} \sum_{i \in \beta_{E}} E_{i}(t)\left(\hat{\boldsymbol{r}} \cdot \boldsymbol{\Theta}_{i}, Y_{n m}\right)_{\mathcal{B}} \boldsymbol{e}_{n},
\end{aligned}
$$

with the initial conditions $\Psi_{n m}^{E}(0)=0$ and $\Psi_{n m}^{H}(0)=0$.

For the numerical integration of (4.14) one can use any suitable explicit time-marching scheme, such as the leapfrog method. However, for the integration of (4.17) and (4.18) we recommend an implicit method such as the trapezoidal rule ${ }^{25}$ since all the eigenvalues of the matrices $\boldsymbol{A}_{n}$ lie in the left half of the complex plane ${ }^{3}$. See also section 5.

\section{The finite-difference time-domain method}

We shall now show how the nonreflecting boundary condition fits into the finite-difference time-domain method (FDTD). First proposed by Yee ${ }^{16}$, this popular method staggers both 
$\boldsymbol{E}$ and $\boldsymbol{H}$ in time and space, and thereby achieves second order accuracy using current values only. Due to the nature of the Yee scheme, the boundary condition is needed only for one of the two electromagnetic field components. We choose to apply it to $\boldsymbol{E}$. Thus $\boldsymbol{E}^{\mathrm{tan}}$ is known at $r=R-\Delta r$ and $r=R$, whereas $\boldsymbol{H}^{\text {tan }}$ is known at $r=R-\Delta r / 2$. The boundary condition is necessary to advance $\boldsymbol{E}^{\tan }$ at $r=R$, since Maxwell's equations (2.1) would require radial derivatives of $\boldsymbol{H}^{\text {tan }}$, whose finite difference approximation involves unknown values of $\boldsymbol{H}^{\text {tan }}$ outside $\mathcal{B}$. Thus we shall use (2.6) to advance $\boldsymbol{E}^{\text {tan }}$ at $r=R$ from time $t$ to time $t+\Delta t$. To do so, we apply (2.6) at $t=t+\Delta t / 2$ and $r=R-\Delta r / 2$, and approximate the first order derivatives on the left by centered finite differences ${ }^{12,24}$.

The right side of (2.6) involves infinite sums, which are truncated at a finite value $N$. It requires the values of $\boldsymbol{\psi}_{n m}^{E}(t)$ and $\boldsymbol{\psi}_{n m}^{H}(t)$ at $t=t+\Delta t / 2$. These are computed concurrently with the solution inside $\Omega$, using the linear ordinary differential equations (2.7) and (2.8). The inner products in (2.7) and (2.8) are computed over the sphere $r=R-\Delta r / 2$ using the fourth order Simpson rule. To solve (2.7) and (2.8) numerically, we opt for the trapezoidal rule $^{25}$, because the eigenvalues of the matrices $\boldsymbol{A}_{n}$ lie in the left half of the complex plane ${ }^{3}$. Since the trapezoidal rule is unconditionally stable, there is no restriction on the time-step in the integration of (2.7) and (2.8). The work required in solving the linear systems (2.7) and (2.8) is negligible, because the matrices $\boldsymbol{A}_{n}$ are very small and remain constant. The trapezoidal rule approximation of $(2.7)$ is

$$
\left(I-\frac{\Delta t}{2} \boldsymbol{A}_{n}\right) \boldsymbol{\psi}_{n m}^{E}\left(t_{k+1 / 2}\right)=\left(I+\frac{\Delta t}{2} \boldsymbol{A}_{n}\right) \boldsymbol{\psi}_{n m}^{E}\left(t_{k-1 / 2}\right)+\Delta t\left(\left.\boldsymbol{E}^{k}\right|_{R-\Delta r / 2}, \boldsymbol{V}_{n m}\right) \boldsymbol{e}_{n}
$$

where $\boldsymbol{E}^{k}$ at $r=R-\Delta r / 2$ is the average of $\boldsymbol{E}^{k}$ at $r=R-\Delta r$ and $r=R$. The trapezoidal rule approximation of $(2.8)$ is

$$
\begin{aligned}
\left(I-\frac{\Delta t}{2} \boldsymbol{A}_{n}\right) \boldsymbol{\psi}_{n m}^{H}\left(t_{k+1 / 2}\right) & =\left(I+\frac{\Delta t}{2} \boldsymbol{A}_{n}\right) \boldsymbol{\psi}_{n m}^{H}\left(t_{k-1 / 2}\right) \\
& +\frac{\Delta t}{2}\left(\left.\boldsymbol{H}^{k-1 / 2}\right|_{R-\Delta r / 2}+\left.\boldsymbol{H}^{k+1 / 2}\right|_{R-\Delta r / 2}, \boldsymbol{V}_{n m}\right) \boldsymbol{e}_{n} .
\end{aligned}
$$

The complete algorithm proceeds as follows:

0 . Initialize $\boldsymbol{E}$ at $t=0$ and $\boldsymbol{H}$ at $t=\Delta t / 2$, and set $\boldsymbol{\psi}_{n m}^{E}=0$ and $\boldsymbol{\psi}_{n m}^{H}=0$ at $t=\Delta t / 2$.

1. Compute $\boldsymbol{E}$ at $t_{k}=t_{k-1}+\Delta t$ at all inner points of $\Omega$ using (2.1).

2. Compute $\boldsymbol{E}^{\mathrm{tan}}$ at $t_{k}$ and $r=R$ using (2.6) applied at $r=R-\Delta r / 2$ and $t_{k-1 / 2}=$ $t_{k-1}+\Delta t / 2$.

3. Compute $\boldsymbol{H}$ at $t_{k+1 / 2}$ using (2.1).

4. Compute $\boldsymbol{\psi}_{n m}^{E}$ and $\boldsymbol{\psi}_{n m}^{H}$ at $t_{k+1 / 2}$ using (5.1) and (5.2), respectively, and return to 1 .

Although the artificial boundary must be spherical, the boundary condition is not tied to any coordinate system, and the grid used inside $\Omega$ can be arbitrary. See Reference 26 for how to fit a Cartesian mesh to curvilinear coordinates, or Reference 27 for a structured spherical mesh without singularities. 


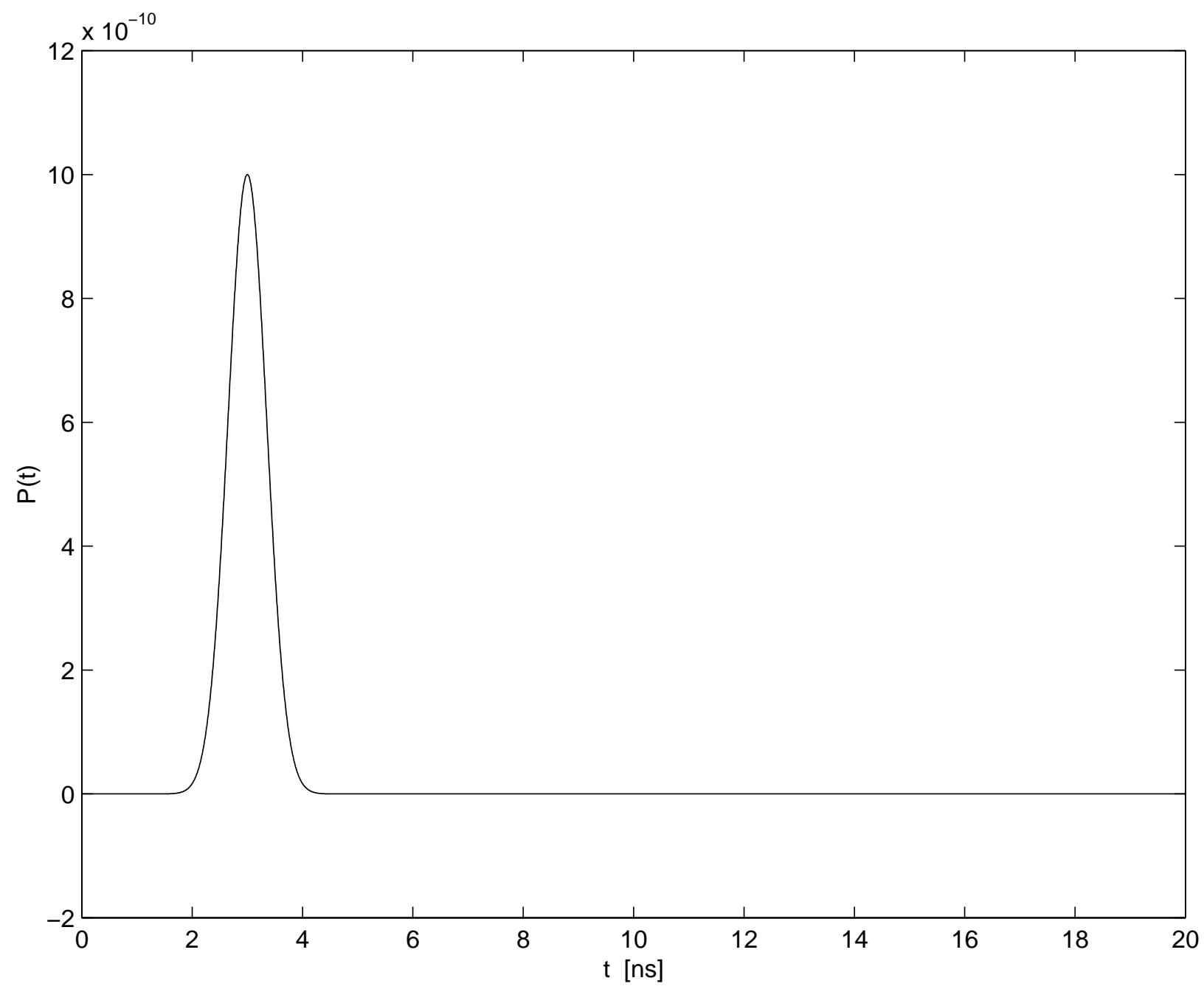

Figure 1: The time dependence $P(t)$ of the dipole source.

\section{$6 \quad$ Numerical results}

We shall now combine the finite-difference time-domain method with the nonreflecting boundary condition, as described in section 3. As a model problem we choose to compute the scattered field of a perfectly conducting sphere of radius $r_{0}$ illuminated by a nearby Hertzian dipole.

The radiating electric dipole is located outside the spherical obstacle at $S=\left(0,0, z_{0}\right)$, $z_{0}>r_{0}$, at distance $z_{0}$ from the origin. It is aligned along $z$, so that its moment points along the positive $z$-axis. Its time dependence, shown in figure 1, is a Gaussian pulse centered about $t=t_{0}$ :

$$
P(t)= \begin{cases}0 & t<0 \\ \alpha e^{-\left(t-t_{0}\right)^{2} / \sigma^{2}} & 0 \leq t \leq 2 t_{0} \\ 0 & t>2 t_{0}\end{cases}
$$



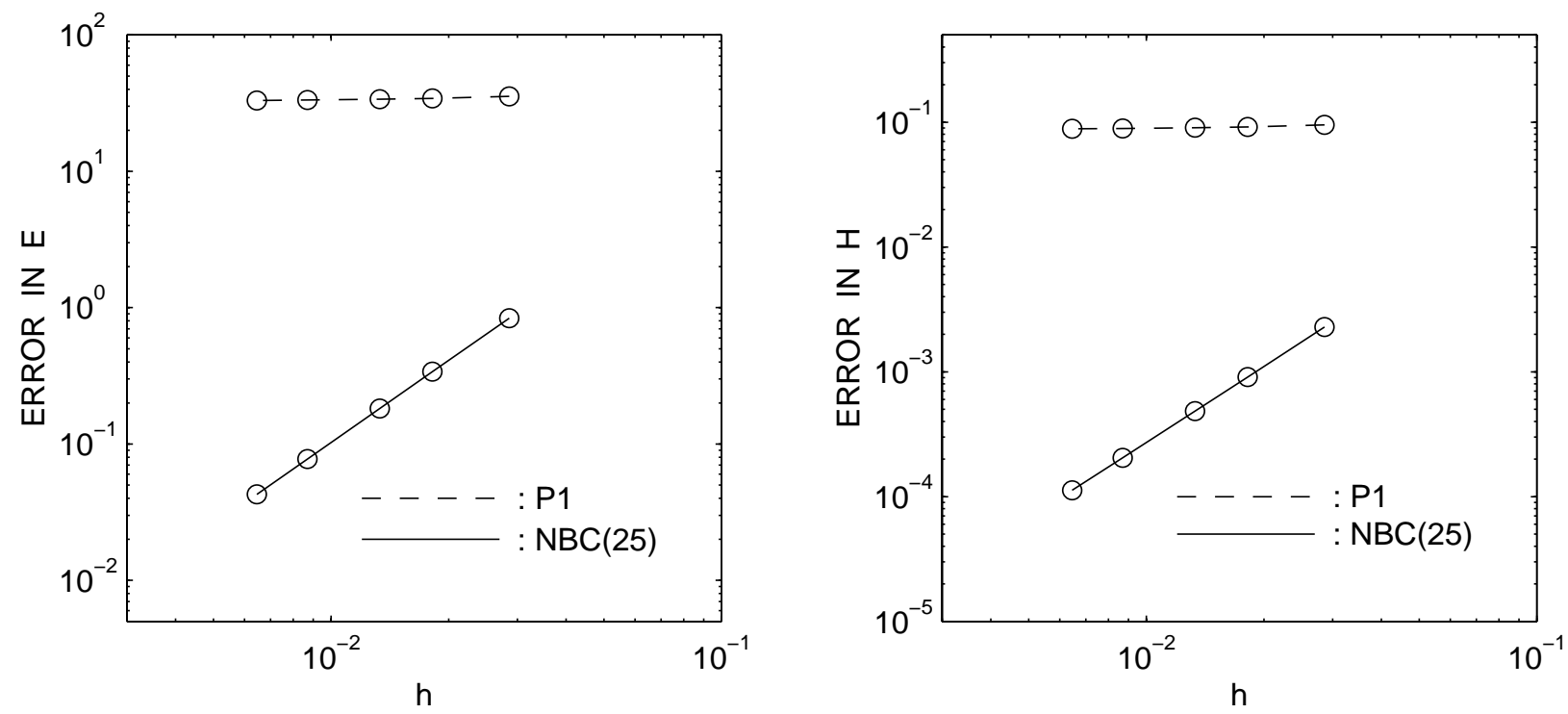

Figure 3: The maximal error in the $L_{2}$ norm over the time interval $[0,20]$ nanoseconds is shown versus the mesh parameter $h=\Delta r: \boldsymbol{E}^{h}$ (left) and $\boldsymbol{H}^{h}$ (right).

exact solution. To compute it inside $\Omega$ we use a much larger domain which extends as far as $r=3.7[\mathrm{~m}]$. This enables us to compute the solution of the initial-boundary value problem in the infinite region outside $\Gamma$ for $0 \leq t \leq 20$ [ns].

In figure 3, we check the accuracy of our numerical method. The maximal error in the $L_{2^{-}}$ norm over the time interval [0,20] nanoseconds is shown versus the mesh parameter $h=\Delta r$, for the following sequence of meshes: $8 \times 88,12 \times 132,16 \times 176,24 \times 264$, and $32 \times 352$. We observe the expected second order convergence of the the full scheme using $\mathrm{NBC}(25)$ as the mesh is refined. This indicates that setting $N=25$ ensures that the error introduced at the artificial boundary is smaller than that of the numerical scheme. However, the error in the numerical solution obtained with P1 does not decrease as the mesh is refined, indicating that the error introduced by using P1 dominates the computation. Indeed, the numerical solution does not converge to the solution of the original problem, but instead converges to the solution of a different problem with $\mathrm{P} 1$ imposed at $\mathcal{B}$. To reduce the amount of spurious reflection at $\mathcal{B}$ one would need to increase the size of $\Omega$. In contrast for the exact boundary condition, $\mathrm{NBC}(20), N$ can be chosen large enough to reduce the error introduced at $\mathcal{B}$ below the discretization error of the numerical method inside $\Omega$, without moving the artificial boundary farther away from the scatterer.

Next, we compare the numerical solutions, obtained using P1 and NBC(20), with the exact solution at two different locations inside $\Omega$ at $r=0.6[\mathrm{~m}]: Q_{1}\left(\theta=45^{\circ}\right)$ and $Q_{2}$ $\left(\theta=170^{\circ}\right)$. The inner and outer radii remain at their current locations $r_{0}=0.5[\mathrm{~m}]$ and $R=0.7[\mathrm{~m}]$, and we use our finest $32 \times 352$ mesh inside $\Omega$.

In figure 4 , the $\phi$-component of the magnetic field is shown at the first location $Q_{1}$. The numerical solution obtained with $\mathrm{NBC}(20)$ is hardly distinguishable from the exact 


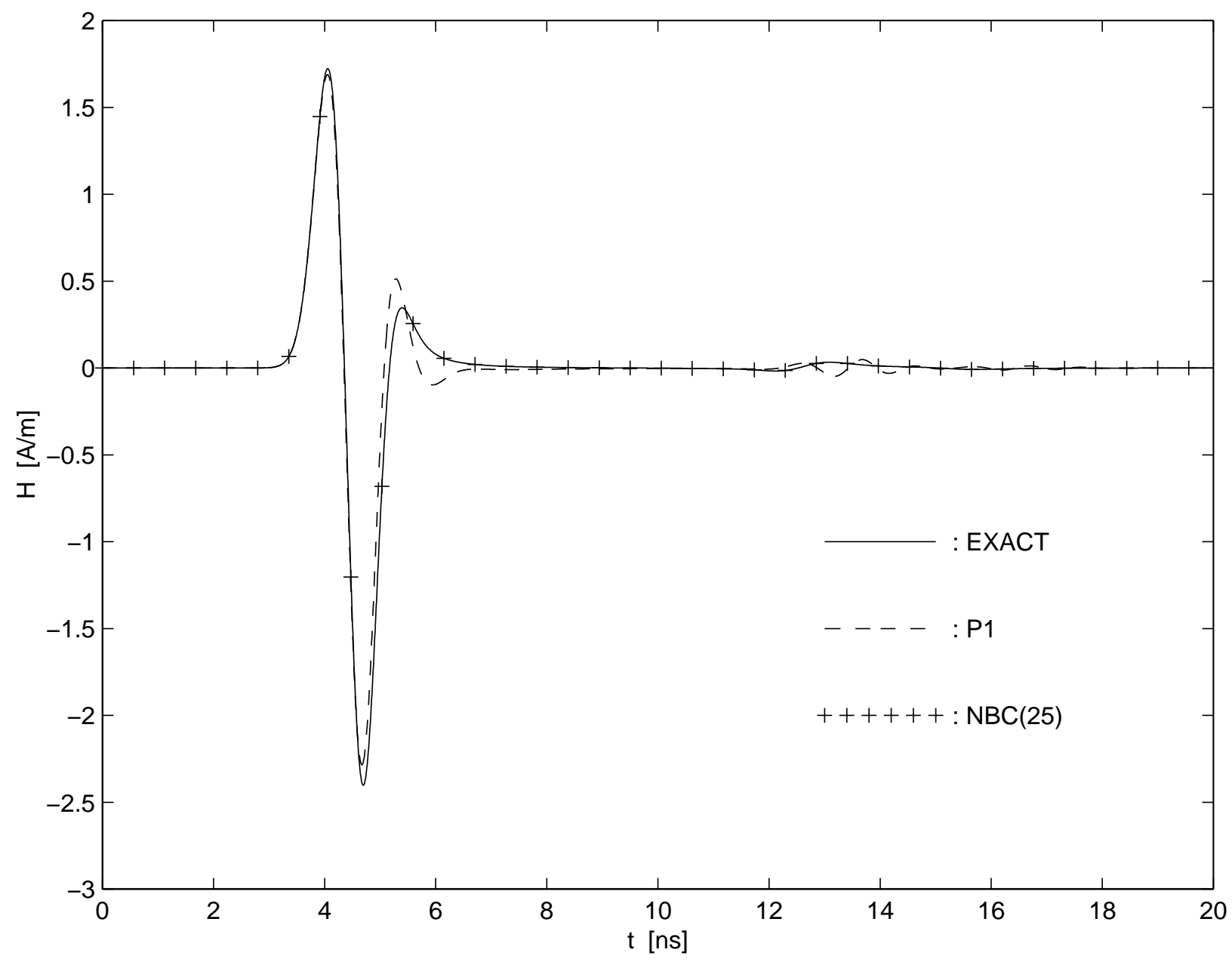

Figure 4: The numerical solutions for $H^{\phi}$, computed using the boundary conditions P1 and $\operatorname{NBC}(25)$, are compared with the exact solution at $Q_{1}$.

solution. While the relative error due to the P1 boundary condition is only a few percent, this seemingly accurate behavior is deceptive. Indeed these locally small reflections travel back into the computational domain, and contaminate the solution everywhere inside $\Omega$, in particular in regions where the solution is of lesser magnitude. To demonstrate this point, we select the next location behind the obstacle at $Q_{2}$, where the electromagnetic field is much weaker. The $\phi$-component of the magnetic field at $Q_{2}$ is shown in figure 5 , and again it agrees completely with the numerical solution obtained using $\mathrm{NBC}(25)$. The solution obtained using P1 agrees with the exact solution for a finite time. It then diverges from it, as the spurious reflection due to the imposition of $\mathrm{P} 1$ reaches this location. Since these spurious reflections due to the $\mathrm{P} 1$ boundary condition are larger than the true solution, the numerical solution with $\mathrm{P} 1$ imposed at $\mathcal{B}$ is meaningless in the shadow region of the sphere.

Finally, we check the long-time accuracy of our method and focus on the second wave packet which arrives at $Q_{1}$ about 10 [ns] later than the first after traveling counter-clockwise 


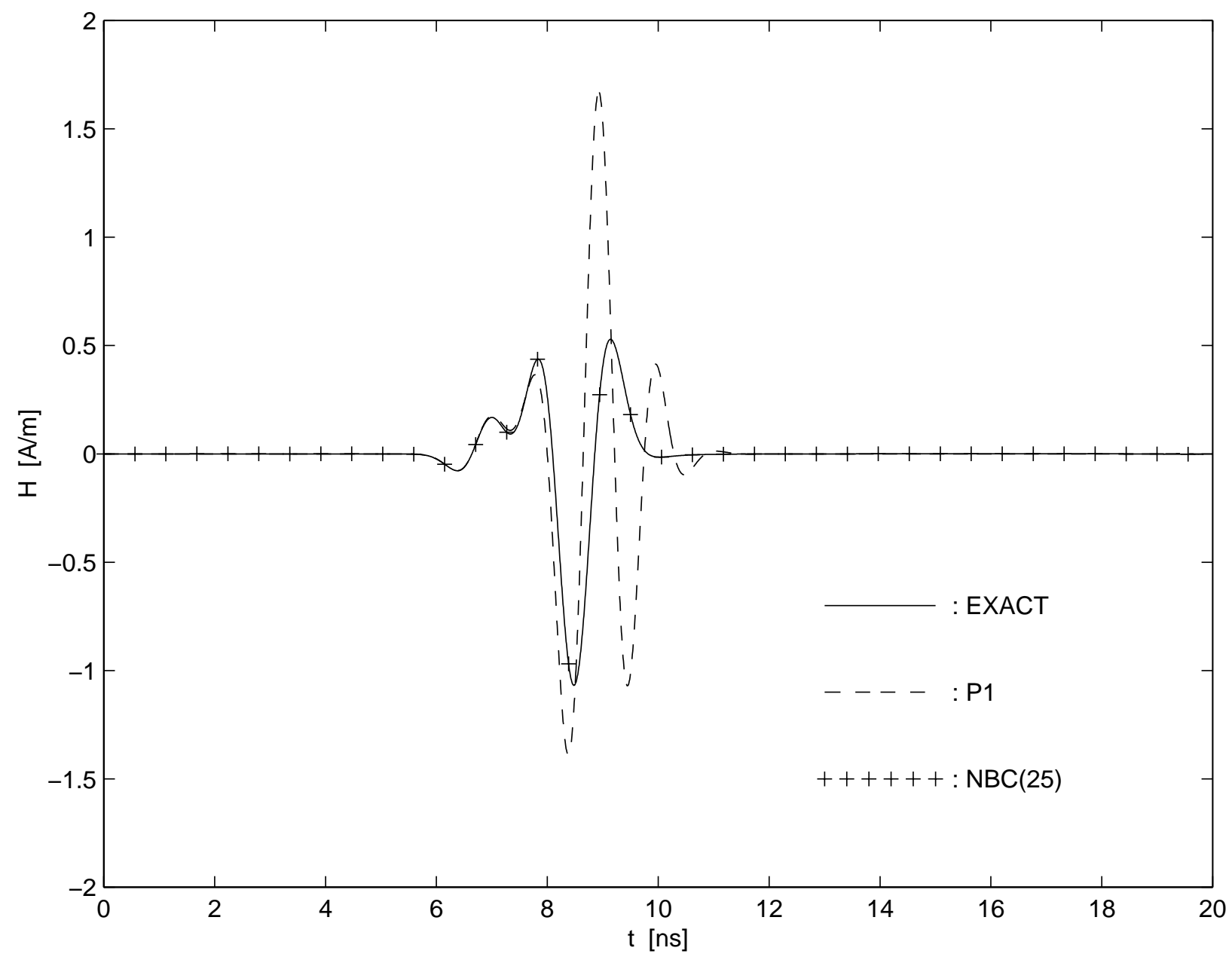

Figure 5: The numerical solutions for $H^{\phi}$, computed using the boundary conditions P1 and $\operatorname{NCB}(25)$, are compared with the exact solution at $Q_{2}$.

around the entire sphere. At this stage the main part of the scattered field has left $\Omega$ and the remaining part is of much smaller amplitude. In figure 6 we show again the $\phi$-component of the magnetic field at $Q_{1}$, yet we strongly magnify the scale to concentrate on the second arrival time. Even after an entire trip around the sphere, the scattered field obtained using $\mathrm{NBC}(25)$ agrees perfectly with that in the unbounded region. In contrast, the local boundary operator P1 has generated multiple spurious reflections which bounce back and forth between the sphere and the artificial boundary, and completely dominate the numerical solution.

\section{Conclusion}

The exact nonreflecting boundary condition (2.6) or (3.26) has been found to be very accurate in numerical computations. It involves only first-order derivatives of the solution, which 


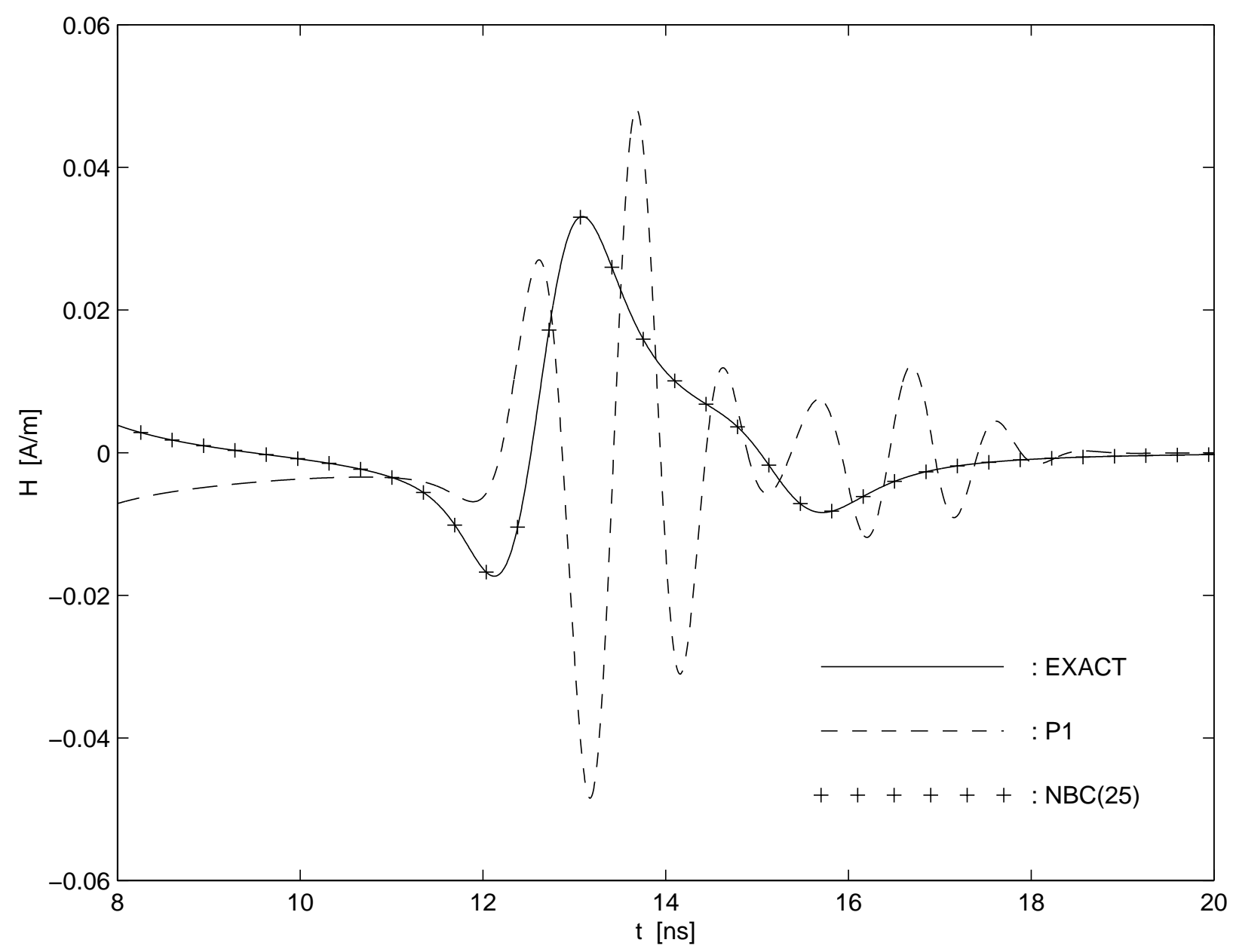

Figure 6: The numerical solutions for $H^{\phi}$, computed using the boundary conditions P1 and $\operatorname{NCB}(25)$, are compared with the exact solution at $Q_{1}$.

makes it robust and easy to use. The boundary condition fits naturally into the finitedifference time-domain method and allows the artificial boundary to be brought as close as desired to the scatterer. It is easy to implement and requires little extra storage and computer time. The alternative formulation (4.11) does not involve any derivatives of $\boldsymbol{E}$ or $\boldsymbol{H}$ and thus fits easily into the variational formulation of Maxwell's equations; hence it is well-suited for use with the finite element method. Although the formulation is global over the artificial boundary, it is explicit and does not require the solution of any large linear system. It only requires inner products with spherical harmonics of the solution on the artificial boundary. With the exact boundary condition the overall numerical scheme retains its optimal rate of convergence, as the error introduced at the artificial boundary can always be reduced below the discretization error due to the numerical method in the interior computational domain. 


\section{Acknowledgement}

I would like to thank Joseph Keller for his helpful comments and suggestions.

\section{References}

1. M. J. Grote and J. B. Keller, "Nonreflecting boundary conditions for Maxwell's Equations", J. Comput. Phys., 139, 327-324 (1998).

2. M. J. Grote and J. B. Keller, "Exact nonreflecting boundary conditions for the time dependent wave equation", SIAM J. Appl. Math., 55, 280-297 (1995).

3. M. J. Grote and J. B. Keller, "Nonreflecting boundary conditions for time dependent scattering", J. Comput. Phys., 127, 52-65 (1996).

4. G. Mur, "Absorbing boundary conditions for the finite-difference approximation of the time-domain electromagnetic-field equations", IEEE Trans. Electromagn. Compat., EMC-23, 377-382 (1981).

5. A. F. Peterson, "Absorbing boundary conditions for the vector wave equation", $M i$ crowave and Optical Techn. Letters, 1, 62-64, (1988).

6. B. Engquist and A. Majda, "Absorbing boundary conditions for the numerical simulation of waves", Math. Comp., 31, 629-651 (1977).

7. A. Bayliss and E. Turkel, "Radiation boundary conditions for wave-like equations", Comm. Pure Appl. Math., 33, 707-725 (1980).

8. R. L. Higdon, "Absorbing boundary conditions for acoustic and elastic wave equations in stratified media", J. Comput. Phys., 101, 386-418 (1992).

9. O. M. Ramahi, "Complementary boundary operators for wave propagation problems", J. Comput. Phys., 133, 113-128 (1997).

10. J.-P. Bérenger, "A perfectly matched layer for the absorbtion of electromagnetic waves", J. Comput. Phys., 114, 185-200 (1994).

11. F. Collino and P. Monk, "The perfectly matched layer in curvilinear coordinates", SIAM J. Scient. Computing, 19, 2061-2090 (1998).

12. A. Taflove, Computational Electrodynamics: The Finite-Difference Time-Domain Method, Artech House, 1995.

13. N. V. Kantartzis, P. G. Petropoulos and T. D. Tsiboukis, "A Comparison of the GroteKeller Exact ABC and the Well-Posed PML for Maxwell's Equations in Spherical Coordinates", in Proceedings of the 14th Annual Review of Progress in Applied Computational Electromagnetics, II, 623-630 (1998). 
14. L. Ting and M. J. Miksis, "Exact boundary conditions for scattering problems", $J$. Acoust. Soc. Amer., 80, 1825-1827 (1986).

15. J. De Moerloose and D. De Zutter, "Surface integral representation radiation boundary condition for the FDTD method", IEEE Trans. Antenn. Propag., 41, 890-895 (1993).

16. K. S. Yee, "Numerical solution of initial boundary value problems involving Maxwell's equations in isotropic media", IEEE Trans. Antennas Propag., AP-14, 302-307 (1966).

17. D. Colton and R. Kress, Inverse Acoustic and Electromagnetic Scattering Theory, Springer Verlag, 1992.

18. J. D. Jackson, Classical Electrodynamics, 2nd edition, John Wiley \& Sons, 1975.

19. C. H. Wilcox, "An expansion theorem for electromagnetic fields", Comm. Pure Appl. Math., 9, 115-134 (1956).

20. M. J. Mohlenkamp, A Fast Transform for Spherical Harmonics, Ph.D. thesis, Dept. of Mathematics, Yale University, 1997.

21. B. Alpert, L. Greengard, and T. Hagstrom, "Rapid evaluation of nonreflecting boundary kernels for time-domain wave propagation", Courant Mathematics and Computing Laboratory report, NYU, 98-004, 1998.

22. R. L. Lee and N. K. Madsen, "A mixed finite element formulation for Maxwell's equations in the time domain", J. Comput. Phys., 88, 284-304 (1990).

23. P. Monk, "A comparison of three mixed methods for the time-dependent Maxwell's equations", SIAM J. Sci. Stat. Comput., 13, 1097-1122 (1992).

24. K. S. Kunz and R. J. Luebbers, Finite Difference Time Domain Methods for Electromagnetics, CRC Press, 1993.

25. E. Hairer, S. P. Nørsett, G. Wanner, Solving Ordinary Differential Equations I, Springer Verlag, 1987.

26. A. Taflove and K. R. Umashankar, "The finite-difference time-domain method for numerical modeling of electromagnetic wave interactions", Electromagnetics, 10, 105126 (1990).

27. C. Ronchi, R. Iacono, P. S. Paolucci, "The 'cubed sphere': a new method for the solution of partial differential equations in spherical geometry", J. Comput. Phys., 124, 93-114 (1996).

28. D. S. Jones, The Theory of Electromagnetism, Pergamon/Macmillan, New York, 1964.

29. R. Holland, "THREDS: A finite-difference time-domain EMP code in 3D spherical coordinates", IEEE Trans. Nucl. Science, NS-30, 4592-4595 (1983). 